\title{
Comunication Skill and Consent Form in Orthodontics
}

\author{
Hussam M Abdelkader* \\ Professor of Orthodontics, Alazhar University, Cairo, Egypt
}

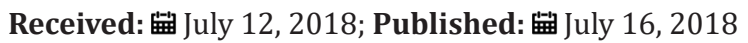

*Corresponding author: Hussam M Abdelkader, Professor of Orthodontics, Alazhar University, Cairo, Egypt

\section{Communication Skill in Orthodontics}

The clinicians' professional communication skill through the process of orthodontic treatment negotiation, simplicity in explaining the case and the proposed orthodontic treatment plan in the simplest direct words away from the hard-technical terms, respecting the other side point of view, as well as, their dignity forms the main corm of successful treatment.

Effective communication is all about conveying your messages to other people clearly and unambiguously. It's also about receiving information that others are sending to you, with as little distortion as possible. Doing this involves effort from both the sender of the message and the receiver. And it's a process that can be fraught with error, with messages muddled by the sender, or misinterpreted by the recipient. When this isn't detected, it can cause tremendous confusion, wasted effort and missed opportunity. In fact, communication is only successful when both the sender and the receiver understand the same information as a result of the communication. By successfully getting your message across, you convey your thoughts and ideas effectively. When not successful, the thoughts and ideas that you actually send do not necessarily reflect what you think, causing a communications breakdown and creating roadblocks that stand in the way of your goals - both personally and professionally.

The first skill that you'll learn; is 'How to Make a Great First Impression": This is essential if you're going to have the chance to commence your message. It is found that your positive impact on the others dependant on

a) $55 \%$ of impact is determined by body language; postures, gestures, and eye contact,

b) $38 \%$ of impact is determined by the tone of your voice.

c) $7 \%$ of impact by the content or the words spooked.

\section{The Seven Keys to Communication Skill}

Communication skill with the others is a very vital and critical issue to be considered in our daily life what so ever our specialty is. Communication skill is based on three fundamental keys arise from them another four keys equally essential and equally vital. These keys are:

a) Key 1: Good understanding of the other b) Key 2: Respecting the other

c) Key 3: Have and find excuse for the other

These three keys must be applied irrespective of:

d) Keys 4: The social level of the other

e) Keys 5: The economic level of the other

f) Keys 6: The education level of the other

g) Keys 7: The physical and mental level of the other

\section{The Computer Imaging Program}

The computer imaging program is good, as long as, it is used within its limitation as an orthodontic treatment aid in addition to the other diagnostic aids. It could make the communication easier, but it must be clear from the very beginning that our treatment outcome will be within the range of the illustrated computer image and not exactly the same. On the other hand, the patients' side should have almost the total responsibility to determine and accept or refute what orthodontic treatment will be performed in view of very clear vision from the orthodontist side.

\section{Signed Consent form in Orthodontics}

Is a signed consent form, form an essential document before commencing orthodontic treatment? And what points it should cover? The answer is yes. From the medicolegal prospective the following points are far enough to satisfy the requirements of the medicolegal prospective:

a) The consent must be written in sample clear direct words in such a way that the proposed treatment and the associated risk if any must be clearly understood by the patient's side whatever their socio-educational level are.

b) The orthodontist must be dead sure that all raised points are clearly understood by the patients' side without any bias.

c) Extraction of any tooth/teeth, if any, is vital point to be considered in the consent form. It must be very clear which tooth/teeth will be extracted in the proposed orthodontic treatment plan, and to clarify that the extraction space(s) will be completely used in favor of approaching the assigned treatment outcome. 
d) To clarify that orthodontic treatment will have two phases; active phase and retentive phase and to stress on the importance of retention as an essential and vital part of the treatment protocol.

e) To clarify to the patients' side any camouflaging treatment which could be undertaken as the last choice and any long-term risk of that treatment protocol if any.

f) Respecting the appointment schedule and if there will be any banality for the broken appointments and/or the orthodontic appliance.

g) Broken appointments, broken appliance(s) or ignorance of the oral hygiene measures to the extent that it could adversely affects the progress of the proposed orthodontic treatment, the clinician will have the right to terminate treatment after written notice to the patients' side, and how to estimate the finical coast at that stage.

h) Treatment total cost must be included and the amount and time of each installment.

\section{ISSN: 2574-1241}

DOI: 10.26717/BJSTR.2018.06.001419

Hussam M Abdelkader. Biomed J Sci \& Tech Res

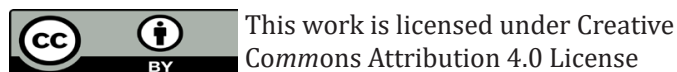

Submission Link: https://biomedres.us/submit-manuscript.php i) To clarify for the patient's side that, the diagnostic records especially facial photographs are to be used only for the benefit of the patient, and if they will be used in a published work, presented in scientific conference or marketing, prior written permission from the patients' side should be taken.

j) The patients' side has the right to terminate orthodontic treatment on their own responsibility at any time without any responsibility on the clinician's side and how to estimate the finical coast at that stage.

k) The policy of patients transfers to other clinicians on the patients' side request, without any responsibility on the clinicians' side, the necessary documents given and how to estimate the finical coast at that stage.

l) A representative of the patient's side should be present at the scheduled visits.

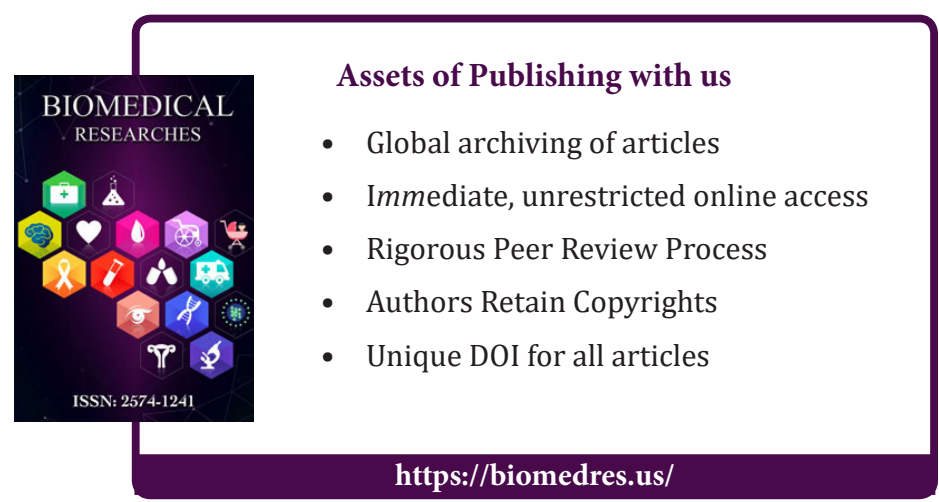

